\title{
Sciatic nerve dependent schwannoma
}

\begin{abstract}
Schwannoma is a tumor composed of Schwann cells. This is the most common benign tumor of the peripheral nerve sheath, representing the $8 \%$ of all soft tissue tumors, affecting the sciatic nerve in less than $1 \%$ of cases. Due to its location in the nerve sheath, these types of tumors produce symptoms related to nerve function and compression of adjacent structures. Diagnosis is made by magnetic resonance and is confirmed by pathological anatomy, often after screening of radicular pathology with normal magnetic resonance and symptomatology compatible with clinical sciatica.

We report the case of a 53-year-old woman with a tumor in the posterior area of the right thigh of one year of evolution. After the examination and the appropriate complementary tests, she was diagnosed with schwannoma of the peripheral sheath of the right sciatic nerve. The patient underwent surgery for tumor exeresis with satisfactory results and acceptable aftermath.
\end{abstract}

Volume I2 Issue 5 - 2020

\author{
Jesús García Álvarez,' Sergio Pérez Ortiz, \\ José Manuel Pelayo de Tomás' \\ 'Department of Orthopedic Surgery and Traumatology, Hospital \\ Universitario Doctor Peset, Spain \\ ${ }^{2}$ Department of Orthopedic Surgery and Traumatology, Hospital \\ Intermutual de Levante, Spain
}

\section{Correspondence: Jesús García Alvarez, Orthopedic Surgery and Traumatology Department, Hospital Universitario Doctor Peset, Av. Gaspar Aguilar, 90. 460I7,Valencia, Spain, \\ Email chusgar256@gmail.com}

Received:September 10,2020| Published: October 30, 2020

Keywords: tumor, schwannoma, sciatica, sciatic nerve

Abbreviations: MR, magnetic resonance; PA, pathological anatomy; CT,computed tomography; EMG,electromyogram

\section{Introduction}

Pain with radicular characteristics is one of the most frequently consulted pathologies in traumatology services, especially pain at the level of the roots that correspond to the sciatic nerve. Although the main cause of this problem is the discopathy that produces compression of the nerve root, there are other pathologies that, although less frequent, we must take into account, such as injuries that affect the peripheral nerve.

We report the case of a patient with radicular type pain in the territory of the sciatic nerve with normal lumbar magnetic resonance. We emphasize the importance of a correct physical examination, and the extension of the study beyond the proximal zone of the nerve root when the most common pathologies are ruled out.

\section{Case report}

We report the case of a 53-year-old Caucasian female patient who came to our center with a palpable tumor in the posterior area of the right thigh with a year of progressive growth, that she relates it to overexertion.

A tumor was palpated in the upper third of the posterior face of the right thigh of approximately $3 \times 5 \mathrm{~cm}$ with hard consistency, adhered to deep tissues, without alterations in the overlying skin and neither vascular nor nervous compromise. She referred spreading pain to the right lower limb and provided recent lumbar magnetic resonance (MR) without pathological findings.

Ultrasound test was requested and revealed a solid mass with well-defined borders, hypoechoic, heterogeneous, with cystic areas, measuring $33 \times 40 \times 55 \mathrm{~mm}$. It was located in the muscular compartment of the biceps femoris with vascularization inside.

We completed the study with MR that reported an ovoid lesion of heterogeneous structure and cystic areas inside, with measurements of $37 \times 33 \times 69 \mathrm{~mm}$ with well-defined borders, maintaining a fatty plane of separation with muscles (signs of non-aggressive lesion), without contrast highlights or signal intensity changes of the muscles adjacent to the lesion (Figure 1). It contacted and displaced the sciatic nerve medially, depending on one of the fascicles of the nerve. With contrast administration, the absorption was progressive, obtaining lowmedium values respect to the arterial peak, being compatible with a nervous sheath tumor.

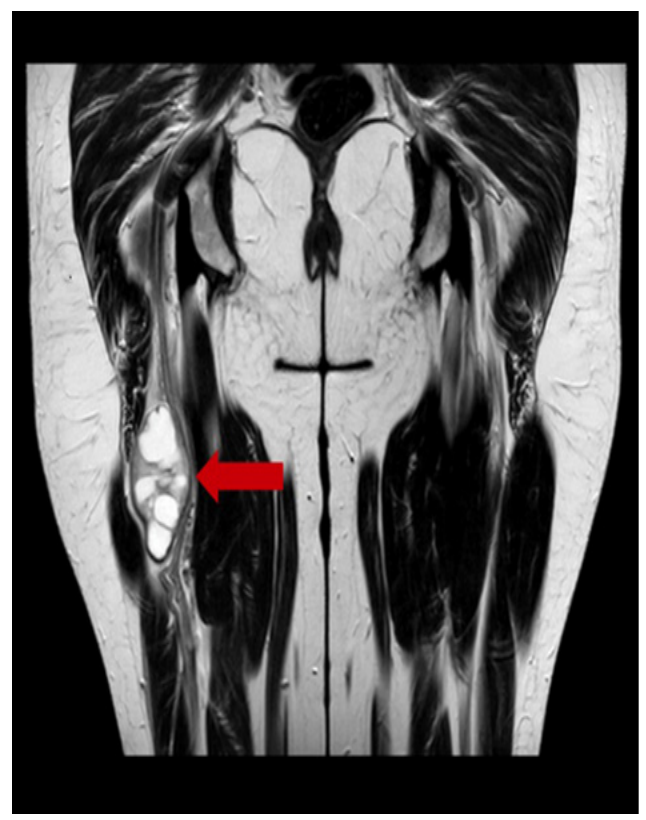

Figure I MR of the lower extremities without contrast, coronal cut: ovoid lesion of heterogeneous structure and dependent areas of one of the fascicles of the sciatic nerve (arrow).

To confirm the diagnosis, a percutaneous biopsy was performed with trucut. The pathological anatomy (PA) report concluded a neural mesenchymal neoplasm (strong and diffuse positivity for S-100 protein) without signs of malignancy, being compatible with a benign tumor of the peripheral nerve sheath (schwannoma) of the right sciatic nerve. 
A complete study was requested that consisted in thoracic, abdominal and pelvic computed tomography (CT) with contrast administration that ruled out systemic extension.

The patient underwent surgery for the exeresis of the tumor. In our case, infragluteal approach was used, pulling away biceps femoris and adductor magnus muscles until the nerve was isolated by plane dissection and neurovascular structures were referenced (Figure 2).

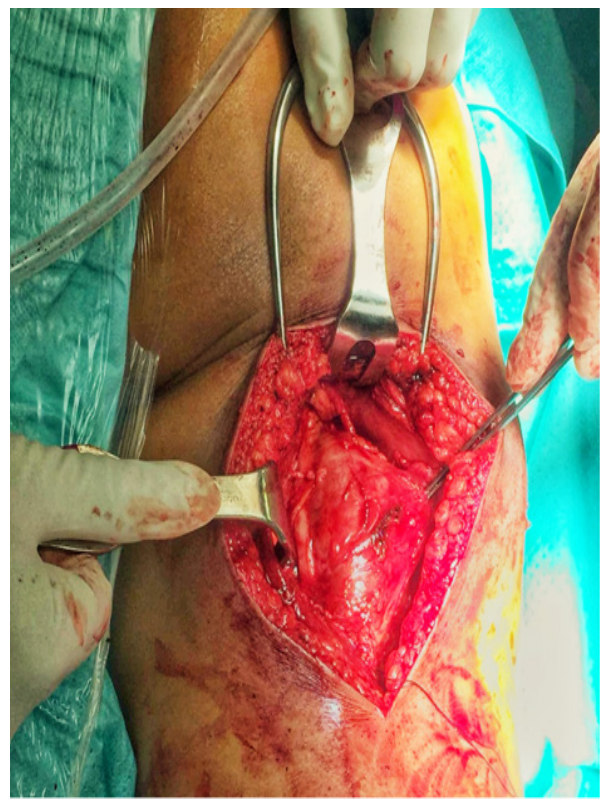

Figure 2 Intraoperative image: nodular tumor with whitish coloration, adjacent to the sciatic nerve in its most cranial portion of the right thigh.

A nodular and whitish-colored tumor was observed next to the sciatic nerve in its most cranial portion of the right thigh. The longitudinal dissection of the peripheral sciatic nerve sheath was performed for complete tumor resection, which was sent to the PA for histological study (Figure 3). The absence of lesion was verified in the proximal and distal ends of the nerve, trying to preserve as many viable nerve fibers as possible.

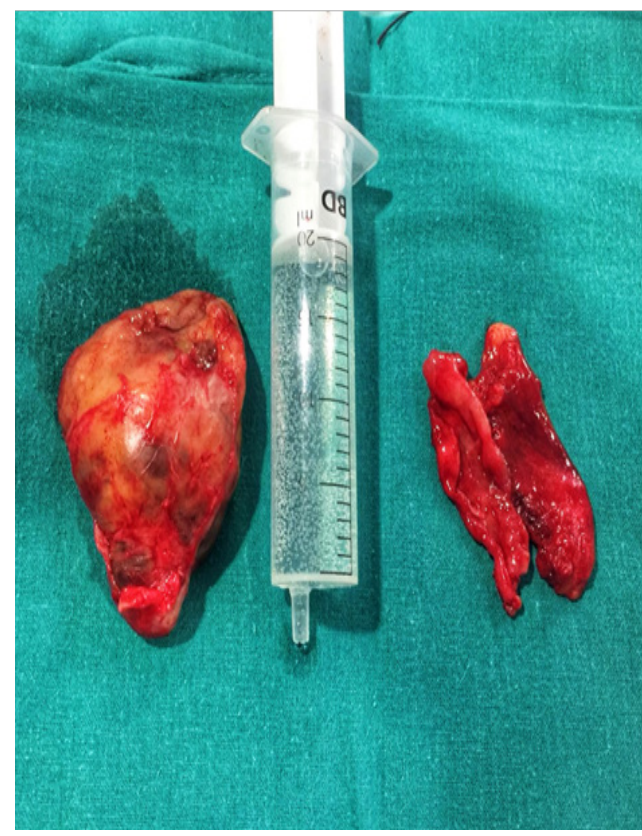

Figure 3 Intraoperative image: excised tumoral piece.
The PA report showed the presence of nodular tissue of mucinous content and with cystic and hemorrhagic areas, without evidence of malignancy, compatible with the diagnosis of schwannoma.In a postoperative re-evaluation of the functional capacity of the operated limb, parenthesis were observed in the dorsal and lateral face of the leg and foot, besides inability to extend the fingers and to flex dorsally the foot. An electromyogram (EMG) was requested to verify the degree of nerve damage, which showed a damage of $95 \%$ of the nerve fibers corresponding to the external popliteal sciatic nerve (EPS) o fibularis communis nerve.

Collaboration was made to the rehabilitation service of our center to design a personal program that allows to reduce the aftermaths to the minimum. Currently, she is carrying out this program.

\section{Discussion}

Schwannoma is a benign tumor derived from the neural sheath of Schwann cells, usually solitary and with a misleading clinical presentation, similar to a radiculopathy and often confused with another radicular pathology. These tumors appear more frequently around the fourth decade of life (63\% between 30 and 59 years), being infrequent in children. They present the same distribution by gender. $^{1-5}$

They present a typical growth along the path of a nervous trunk, growing eccentrically to the nerve, not including its bundles. This fact makes the difference with neurofibroma, giving the schwannoma a different treatment and a much more favorable prognosis. This tumor appears more frequently in the upper limbs, affecting the median

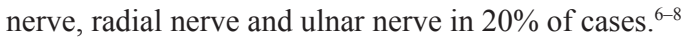

They usually have a misleading clinical presentation, mainly due to a long subclinical course. When they cause symptoms, their most frequent presentation simulate radiculopathy, with sensory alterations, being motor loss a less common presentation, so that it is often confused with other nerve root pathologies, secondary to degenerative problems of the spine. A radicular pain with a long period of evolution, which does not respond to anti-inflammatory treatment and rest, and the lack of a physical examination and imaging tests that are compatible with lumbar pathology, should make us think about peripheral nervous pathology. The palpation of the tumor is usually difficult in many cases due to its location and, if the palpation is possible, indicates a long period of evolution..$^{9-19}$

The diagnosis is usually evident by optical microscopy, although confirmation by immunohistochemical techniques may be useful. It presents two different histological types that may coincide in the same tumor: Antoni type A, with a high density of fusiform cells organized in palisade-shaped; and type B, which presents a pattern of lower cellularity with amorphous material between them. These types of tumors have a strong positivity for the S-100 protein. ${ }^{20,21}$

Despite being benign, some cases of malignant degeneration have been described (less than $0.001 \%$, depending on the series) and especially in cases associated with neurofibromatosis type $1 .^{22,23}$

Its gold standard treatment is the marginal resection of the tumor, preserving the anatomy and functionality of the nerve. This is possible in the case of schwannomas, which grow eccentrically to the tumor, but not in the case of neurofibromas that require much more aggressive surgery. Recurrence is infrequent after tumor excision. ${ }^{24,25}$

\section{Conclusion}

In summary, schwannomas are benign tumors with insidious clinical presentation, which makes their diagnosis difficult. We must 
think about them when faced with a patient with clinical symptoms similar to a radicular pathology, but with physical examination and complementary tests not compatible with this pathology. An early surgical treatment is very important, in order to avoid the possible aftermaths that can cause the compression of neighboring structures and to rule out another tumor with a worse prognosis than schwannoma.

\section{Acknowledgments}

None.

\section{Conflicts of interest}

The authors declare there are no conflicts of interest.

\section{Funding}

None.

\section{References}

1. Evans DG, Bowers NL, Tobi S, et al. Schwannomatosis: a genetic and epidemiological study. JNeurol Neurosurg Psychiatry. 2018;89(11):12151219 .

2. Maes R, Ledoux P, Brouckere G. A rare cause of sciatica: Sciatic nerve schwannoma. Report of one case with long subclinical course and misleading presentation. SICOT-J. 2020;6(16).

3. Maselli F, Testa M. Superficial peroneal nerve schwannoma presenting as lumbar radicular syndrome in a non-competitive runner. $J$ Back Musculoskelet Rehabil. 2019;32(2):361-365.

4. Wang P, Chen $\mathrm{C}$, Xin X, et al. Giant intrapelvic malignant peripheral nerve sheath tumor mimicking disc herniation: A case report. Mol Clin Oncol. 2016;5(5):653-656.

5. Gosk J, Gutkowska O, Urban M, et al. Results of surgical treatment of schwannomas arising from extremities. Biomed Res Int. 2015;547926.

6. Andrzej Wronski K, Frąckowiak L, Mariusz K. Nervio ciático gigante Schwannoma - Reporte de un caso. Chirurgia Polska. 2014;16(2):91-93.

7. Eroglu U, Bozkurt M, Ozates O, et al. Sciatic nerve schwannoma: case report. Turk Neurosurg. 2014;24(1):120-122

8. Desai KI. The surgical management of symptomatic benign peripheral nerve sheath tumors of the neck and extremities: an experience of 442 cases. Neurosurgery. 2017;81(4):568-580.

9. Díaz-Rojas LM, Lorente-Gómez A, Lorente-Moreno R. Tumor gigante de vaina neural. Acta ortop mex. 2016;30(6):320-322.
10. Godkin O, Ellanti P, O'Toole G. Large schwannoma of the sciatic nerve. BMJ Case Rep. 2016;bcr2016217717.

11. Kralick F, Koenigsberg R. Sciatica in a patient with unusual peripheral nerve sheath tumors. Surg Neurol. 2006;66(6): 634-637.

12. Kuntz IV C, Blake L, Britz G, et al. Magnetic resonance neurography of peripheral nerve lesions in the lower extremity: technical report. Neurosurgery. 1996;39(4):750-757.

13. Mezian K, Záhora R, Vacek J, et al. Sciatic Nerve Schwannoma in the Gluteal Region Mimicking Sciatica. Am J Phys Med Rehabil. 2017; 96(7):e139-e140.

14. Madi S, Pandey V, Mannava K, et al. A benign ancient schwannoma of the tibia nerve. BMJ Case Rep. 2016;2016:bcr2016215620.

15. Nawabi DH, Sinisi M. Schwannoma of the posterior tibial nerve. The problem of delay in diagnosis. J Bone Joint Surg Br. 2007;89(6):814-816.

16. Younus A, Kelly A, Lekgwara P. Entrapment neuropathy caused by a schwannoma of the posterior tibial nerve. A case report and literature review. Interdiscip Neurosurg. 2020;21(100780).

17. Laffont M, Michaud M, Pugnet G, et al. Lower limb pain of unusual cause. Rev Med Interne. 2015;36(8):566-567.

18. Mansukhani SA, Butala RR, Shetty SH, et al. Sciatic nerve schwannoma: a case report. J Orthop Surg. 2015;23(2):259-261.

19. Rustagi T, Badve S, Parekh A. Sciatica from a foraminal lumbar root schwannoma: case report and review of literature. Biomed Res Int. $2012 ; 142-143$

20. Artico M, Cervoni L, Wierzbicki V, et al. Benign neural sheath tumours of major nerves: characteristics in 119 surgical cases. Acta Neurochirurgica. 1997;139(12):1108-1116.

21. Blanchard C, Dam-Hieu P, Zagnoli F, et al. Chronic sciatic pain caused by sciatic nerve schwannoma. Rev Med Interne. 2008;29:748- 750.

22. Sarabia JM, Nicolás G, Carrillo FJ. Schwannomas múltiples de nervio mediano: descripción de un caso. Rev esp cir ortop Traumatol. 2009;53(2):120-122.

23. Schulz A, Grafe P, Hagel C, et al. Neuropathies in the setting of Neurofibromatosis tumor syndromes: Complexities and opportunities. Exp Neurol. 2018;299(Pt B):334-344.

24. Dinakar D, Rao SB. Neurilemomas of peripheral nerves. Int Surg. 1971;55(1):15-19.

25. As-Sultany M, Ben-Ghashir N, Mistry A, et al. Giant schwannomas of the sciatic nerve. BMJ Case Rep. 2017;2017. 